\title{
Regulation of Autophagy and Inflammation Through Physical Exercise in Patients with Chronic Kidney Disease: Protective Factor in Individuals Affected by COVID-19
}

\author{
Matheus Ribeiro Bizuti ${ }^{1}$, Júlia Leão Batista Simões ${ }^{1}$, Gabriel Rossi Francisco ${ }^{1}$, \\ Gabrielli Drechsler ${ }^{1}$, Fabiana Brum Haag ${ }^{2}$, Rafael Luiz Pereira ${ }^{3}$ \\ and Débora Tavares de Resendee Silva ${ }^{4^{*}}$
}

\footnotetext{
${ }^{1}$ Department of Medicine, Federal University of Fronteira Sul, Chapecó, SC, Brazil. ${ }^{2}$ Department of Cardiology, Federal University of Fronteira Sul, Chapecó, SC, Brazil. ${ }^{3}$ Department of Physiology, Federal University of Paraná, Curitiba, PR, Brazil. ${ }^{4}$ Department of Pathology, Federal University of Fronteira Sul, Chapecó, SC, Brazil.
}

Authors' contributions

This work was carried out in collaboration among all authors. Authors MRB, FBH, RLP and DTDRS prepared the study design, in the literature review and participated in the writing of the manuscript. Authors JLBS, GRF and GD contributed to the literature review and the writing of the manuscript. All authors read and approved the final manuscript.

Article Information

DOI: 10.9734/ARRB/2021/v36i130334 Editor(s):

(1) 1. Dr. Bechan Sharma, University of Allahabad, India. (2) Dr. Gonzalo Emiliano Aranda Abreu, Veracruzana University, Mexico. (3) Dr. Manikant Tripathi, Dr. Ram Manohar Lohia Avadh University, India. Reviewers: (1) Iskra Ventseslavova Sainova, Bulgarian Academy of Sciences (BAS), Bulgaria. (2) Sebastião David Santos-Filho, Federal University of Rio Grande do Norte, Brazil.

(3) A. B. M. Helal Uddin, International Islamic University Malaysia, Malaysia. Complete Peer review History: http://www.sdiarticle4.com/review-history/66103

Mini-review Article

Received 15 February 2021 Accepted 01 March 2021

Published 15 March 2021

\section{ABSTRACT}

In March 11, 2020, the World Health Organization (WHO) characterized the rapid and uncontrollable spread of the new Coronavirus as a pandemic, given that this disease has high severity and morbidity and mortality. The epicenter of the SARS-CoV-2 outbreak was the city of Wuhan, China. Individuals with associated comorbidities, such as patients with chronic kidney 
disease (CKD), are at increased risk of being affected by the severe form of the disease. In this sense, it is known that people with CKD have a more sedentary lifestyle, with reduced physical exercise. Thus, physical exercise is able to modulate the inflammatory process resulting from CKD, acting as a regulator of the immune system, as well as assisting in the control of renal autophagy. It appears that physical activity contributes to the improvement of the population's quality of life and acts as an effect of disease prevention. Hence, people who live with CKD in times of the pandemic of COVID-19, by adopting physical activity as a life practice, will have the consequence of strengthening the immune system by modulating inflammation and increasing the control of renal autophagy. Therefore, the practice of exercise is suggested when facing COVID-19.

Keywords: Autophagy; chronic kidney disease; exercise; inflammation - SARS-CoV-2.

\section{INTRODUCTION}

A new outbreak of viral pneumonia was reported in Wuhan, capital of Hubei province, China, in December 2019. Named Severe Acute Respiratory Syndrome Coronavirus 2 (SARSCoV-2), coronavirus 2019 (COVID-19) was declared a global public health emergency on January 30, 2020 and a pandemic in March of the same year [1].

The COVID-19 pandemic represents a major challenge to the global health system, as it presents high morbidity and mortality. In some people, COVID-19 manifests itself through acute respiratory distress syndrome (ARDS) and severe pneumonia. However, the most of the patients affected by the disease are asymptomatic or have mild symptoms, such as dry cough, fever, fatigue, dyspnoea and/or myalgia. Individuals with comorbidities, such as chronic kidney disease (CKD), cardiovascular disease (CVD), chronic liver disease, hypertension and diabetes, are at increased risk of being affected by the disease in its most severe form. Recent studies suggest that COVID-19 predisposes to adverse renal events, such as acute kidney injury (AKI) [2]. CKD is characterized by renal dysfunction associated with falls in the glomerular filtration rate (GFR), in the case less than $60 \mathrm{~mL} / \mathrm{min} / 1.73 \mathrm{~m}^{2}$, persisting for a period of at least three months or can be identified by an established histological damage. Renal impairment occurs through different factors, including hypertension, diabetes, drug use, smoking, genetic condition, obesity, among others. In 2017, the number of deaths from complications related to CKD was 1.2 million, corresponding to a percentage of $4.6 \%$ of deaths globally [3].

In order for COVID-19 to evolve, SARS-CoV-2 must interact with the host's immune system in order to influence the immune response against infection. Thus, factors such as age, physical status and nutritional status directly influence the development of COVID-19. Thus, physical activity, when performed regularly, improves the quality of life of the population. As for the immune system, physical exercise reduces the possibility of worsening of systemic inflammatory processes, in order to strengthen immune surveillance [1].

In situations of renal impairment, one of the ways of providing therapies is the regulation of the inflammatory process, given that inflammation is the basis of kidney diseases. Autophagy consists of a system of cellular degradation in order to provide a picture of homeostasis, acting through self-degradation and the reconstruction of damaged organelles and proteins. CKD patients have impaired autophagic systems. Thus, regular physical activity contributes to the adaptation of the immune system and the autophagy process [4].

Therefore, this article aims to conduct an integrative literature review relating the role of physical exercise on the process of cellular autophagy in patients with kidney disease affected by COVID-19. To this end, the bibliographic study included knowledge about chronic kidney disease, autophagy, inflammation and the effects of physical exercise on the immune system in patients with CKD and therefore, proposed the understanding of the most recent information about the interaction between COVID-19 and its renal manifestations, also understanding its relationship with the physical conditions of individuals.

\section{RENAL PATHOPHYSIOLOGY}

The kidneys are fundamental organs for the maintenance of homeostasis of the human body. Thus, the progressive decrease in renal function results in the impairment of essentially all other 
organs. Actual activity is proportional and assessed by glomerular filtration (GF) and its decrease is seen in CKD, which is defined as a pathological abnormality of the kidney, such as hematuria and/or proteinuria, or a reduction in GFR of $<60 \mathrm{~mL} /$ minute/1.73 $\mathrm{m}^{2}$ for more than 3 months [5].

Thus, CKD is defined by the injury to the renal parenchyma and/or by the renal functional decrease during a period equal to or greater than three months. The pathophysiology is complex and regardless of the method of kidney injury, this damage results in a sequence of events. Therefore, it is believed that there is an increase in intraglomerular pressure with glomerular hypertrophy, given that due to the loss of nephrons, the kidney uses this development to maintain constant glomerular filtration [5].

In addition, CKD is classified into five distinct stages based on the estimated glomerular filtration rate (eGFR) and when FG reaches very low values, less than $15 \mathrm{~mL} / \mathrm{min} / 1.73 \mathrm{~m}^{2}$, what we call bankruptcy is established injured renal function, often presenting as fractional flow reverse (FFR), that is, the most advanced stage of progressive functional loss observed in CKD. The new definition of CKD, in use since 2002, allowed the disease to stagnate regardless of its cause. From this new approach, it became evident that CKD is much more frequent than previously considered and its clinical evolution is associated with high rates of morbidity and mortality [5].

In the kidney injury scenario, there is an increase in glomerular permeability for macromolecules, namely: transforming growth factor beta (TGF beta), fatty acids, pro-inflammatory markers of oxidative stress and proteins. Thus, these molecules can generate toxicity to the mesangial matrix, causing expansion of the mesangial cell, inflammation, fibrosis and glomerular scarring, which makes the nephrons less active. Furthermore, this cellular damage positively fuels this cycle, since it increases the production of angiotensin II, which activates the regulation of TGF beta and contributes to collagen synthesis and renal healing within the glomerulus [6].

Therefore, the concomitant structural, molecular, cellular and biochemical changes appear to be responsible for progressive renal scarring and loss of renal function. All forms of CKD are associated with tubulointerstitial disease, although the mechanism of the injury is not known, it is believed to be secondary to a reduction in blood supply, in addition to an infiltration of lymphocytes and inflammatory mediators, which result in fibrosis. interstitial and tubular atrophy [6].

\section{COVID-19 AND CKD}

Direct infection with SARS-CoV-2 occurs through an angiotensin-converting enzyme 2 (ECA2) that acts as a receptor, therefore, any organ that has the ECA2 gene can become a target of the virus. From this perspective, studies have shown that renal tissue has a higher expression of this gene than lung tissue, increasing the affinity of SARSCoV-2 by the kidneys by 10 to 20 times [7]. Confirming the direct cytotoxic effect on renal epithelial cells, studies have found, through immunohistochemistry and in situ hybridization, fragments of the SARS-CoV-2 positive ribonucleic acid polymerase (RNA) gene in renal specimens of patients who died of SARS. It is known that ACE2 is expressed in different renal cells, namely: mesangial cells, podocytes, Bowman's capsule parietal epithelium, collecting ducts and cells of the proximal contorted tubule. The potential direct lesion of the virus was demonstrated by Diao et al. [8] at the necropsy of six patients, who presented the SARS-CoV-2 nucleocapsid protein (NP) in the renal tubule [8].Thus, it can be understood that patients with kidney disease, currently 850 million people worldwide, are more likely to develop complications in the context of the COVID-19 pandemic. Furthermore, considering that CKD causes at least 2.4 million deaths per year and that its mortality rate is increasing, it is necessary to understand how the mechanism of the pathophysiology of CKD can be amplified by viral infection [9].

The involvement of patients with CKD can occur in different ways, resulting in a worsening of the clinical picture; in the development of other pathologies and in increasing levels of mortality. Thus, renal dysfunction may occur due to the direct involvement of the virus and the replication of SARS-CoV-2 in the renal tissue; local change in homeostasis of the renin-angiotensinaldosterone system (RASA) and by increasing the inflammatory process, by adding the lowgrade inflammation of CKD and developing a systemic inflammatory response triggered by the "cytokine storm" $[8,10,11]$.

Defined as a condition with reduced kidney function, CKD has a GFR threshold $<60 \mathrm{ml} \cdot$ min- 
$1 \cdot 1,73 \mathrm{~m}^{2}$ or presence of kidney damage for more than three months, configuring a catabolic state related to protein loss and several disorders resulting from uremia, as well as reduced muscle synthesis in the uremic environment. Therefore, its progression is responsible for increasing mortality and resulting in end-stage renal disease (DRET), which depends on dialysis or transplantation as therapy [12].

Renal microcirculation occurs through a unique network responsible for controlling the corticomedullary osmotic gradient in order to promote the reabsorption of molecules and fluids, as well as excretion of urine at the ideal concentration. Thus, increased levels of proinflammatory cytokines activate endothelial cells and leukocytes, inducing overexpression of proinflammatory factors, reactive oxygen species (ROS), bioactive lipids and adhesion molecules at the site, resulting in the disruption of the glycocalyx, which has an endothelial barrier function, activation of the coagulation system and vasoreactivity $[13,14]$.

Therefore, intra-renal or systemic inflammation triggers a deregulation of the microvascular response to its regulators, resulting in the production of tubular toxins and tubular injury, as well as a decrease in the number of nephrons and onset of CKD. Thereby, it is clear that in $C K D$, oxidative stress and inflammation are closely associated and related to the progression and worsening of CKD. In addition, there is a dysregulation of the immune system which, added to the renal expression of the ECA2 receptor, may explain the vulnerability of patients with CKD to COVID-19, as well as the propensity to develop critical cases [15]. Furthermore, in the cytokine storm, inflammatory exacerbation and damage to the immune system in healthy tissue occurs [16]. This attack is due to the activation of CD8+ and CD56+ (natural killer) T cells, given that the differentiation cluster $68(\mathrm{CD} 68+)$ and macrophage infiltration in the tubules and interstitial tissue and acute tubular necrosis (NTA) severe, was revealed by post-mortem analysis of patients infected with SARS-CoV-2 $[8,17]$. In this way, this inflammation is able to modify, as well as interfere, in the regulation of intrarenal microcirculation and in the distribution of perfusion, contributing to kidney injury and potentiating the progression of CKD [18].

In a cohort study, the combined prevalence of pre-existing CKD and DRET was found to be
$5.2 \%(2.8-8.1)$ and $2.3 \%(1.8-2.8)$ [2]. Thus, it is known that the incidence of patients with known CKD ranged from $0.7-47.6 \%$, depending on the series described. Another aggravation is an increase in mortality, as well as a higher incidence of acute renal failure (ARI) in these patients, since CKD is an independent risk factor for ARI [19-22]. In addition, proteinuria and hematuria have been observed in patients with COVID-19, demonstrating a cytotropic effect of the virus on podocytes, given that protein concentrations in the urine may result from direct podocyte damage to an expression of ECA2 $[16,21,23]$. Thereby, it is understood that renal manifestations due to SARS-CoV-2 infection are associated with an increased risk of severe COVID-19 and fatal outcomes and it has been reported that, in patients with CKD, there is a rate of $83.93 \%$ severity of COVID-19, in addition to a mortality of $53.33 \%[21,24]$.

Systemic inflammation has also been associated with renal neoplasms, especially in terms of prognosis, being also a promoter of cell transformation and metastasis and, considering that the PI3K-AKT-mTOR signaling pathways mediate mechanisms of the pathogenesis of carcinoma, this autophagic pathway can be related to a possible prevention of kidney diseases. Finally, considering that inflammation, malnutrition and loss of protein energy are important contributors to mortality in patients with CKD, exercise can be proposed to reduce this inflammatory process (Fig. 1) [25-28].

CKD patients, when infected with SARS-CoV-2, may suffer direct infection by the virus in the kidney, since this organ has ECA2 receptors in the proximal contorted tubule, the collecting tubule and the nephron (podocytes, mesangial cells and the capsule parietal epithelium) as well as Bowman's capsule. However, there is also renal impairment due to the increased inflammation caused by the "cytokine storm" and a change in homeostasis due to changes in the aldosterone-renin-angiotensin system. Thus, the kidney with CKD that already presents lesions, suffers from an intrarenal inflammation that activates leukocytes (macrophages, monocytes, eosinophils, basophils and neutrophils) and the endothelial cells of the parietal epithelium. This activation raises the levels of proinflammatory cytokines, bioactive lipids and ROS, triggering lesions in the renal barriers that result in lesions in the nephrons and tubules. 


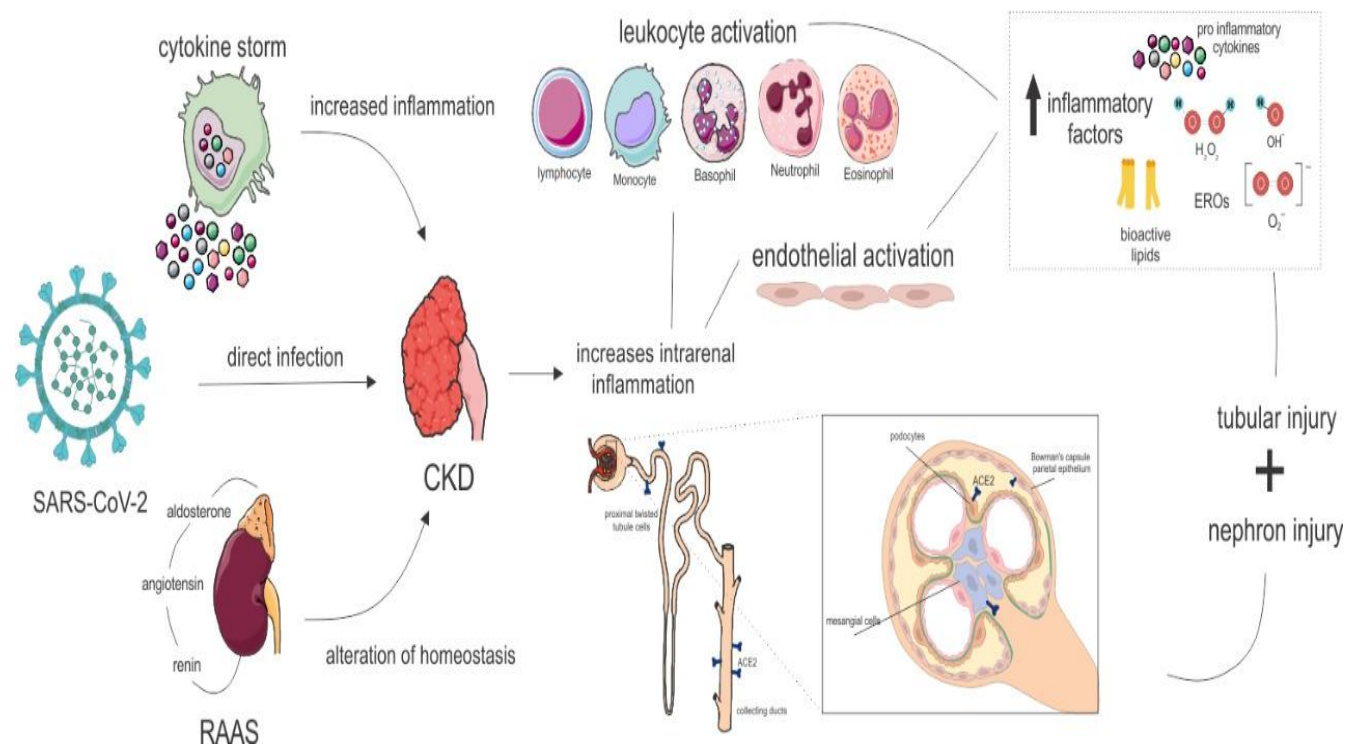

Fig. 1. SARS-CoV-2 performance in CKD patients Source: The authors, 2020

\section{INFLAMMATION AND AUTOPHAGY IN CKD}

CKD is characterized by a local inflammatory process, a fact that can be accentuated in cases of infection by Sars-Cov-2, the causative agent of COVID-19 [29,30]. Thus, in this condition, an increase in the infiltration of M1 macrophages, responsible for pro-inflammatory conditions, in addition to the infiltration of mast cells and leukocytes in the renal interstitium, can occur, which may contribute to a respiratory burst due to a greater oxygen uptake and thus, lead to a greater accumulation of oxygen free radicals, in addition to the release of pro-inflammatory cytokines. This inflammatory condition can be divided in a chronic and acute phase, these high concentrations of pro-inflammatory cytokines are found in the chronic condition of inflammation, both locally and systemically, namely Interleukin1 (IL-1), Interleukin-6 (IL-6) and Tumor Necrosis Factor Alpha (TNF-alpha), the part of the inflammatory condition previously mentioned, with the infiltration of macrophages and leukocytes in the local tissue, as a constituent of the acute condition of inflammation, thus, each inflammatory stage history to be analyzed for study or interventions should consider the inflammatory phase in which it is [31,32]. In addition, CKD can also affect the cell's autophagic function, responsible for cell recycling, which involves processes of reconstruction and self-degradation of defective proteins and organelles [29]. It should also be noted that the renal tubules, which have several mitochondria, due to their high energy demand, need autophagy to maintain these organelles in their normal functioning, however, studies suggest an increase in formation of the autophagosome and the expression of proteins related to autophagy in CKD, that is, an increase in the autophagic process [24,33].

With this increase in the autophagic process, that is, this imbalance, there may be a reduction in the elimination of damaged mitochondria and consequently, a malfunction thereof, which may trigger an increase in the release of ROS. Furthermore, it is important to note that this increase in ROS leads to an even greater increase in the inflammatory response $[29,33]$. These factors combined, trigger a worsening of the inflammatory condition previously mentioned, further complicating the condition. As if the aforementioned relationships were not enough, the increases in ROS are also capable of exerting a modulatory effect on autophagy, this is in a negative way, that is, inhibiting the $\mathrm{PI3K} / \mathrm{AKT} / \mathrm{mTOR}$ pathway, which has the ability to inhibit autophagy, therefore, this generates an even greater increase in autophagy that was already exacerbated [34,35]. The autophagic process consists of several molecules that interact with each other so that autophagy can be performed with precision, namely: AMP-activated Protein Kinase (AMPK) is the target of rapamycin in mammals (mTOR), which has a very important 
role important as part of the phosphoinositide 3kinase $(\mathrm{PI} 3 \mathrm{~K}) /$ serine/threonine kinase $(\mathrm{AKT}) / \mathrm{mTOR}$ or "PI3K/AKT/mTOR" pathway, a vital pathway for cell growth, proliferation, differentiation, survival, motility, metabolism and autophagy [34]. AMPK acts as an autophagypromoting molecule, this is due to its positive regulatory power in UNC-51, similar to ULK1 kinase, one of the key molecules to form the autophagosome. In this sense, the $\mathrm{PI3K} / \mathrm{AKT} / \mathrm{mTOR}$ pathway also has regulatory power over ULK1, but in this case, with a negative effec [34-38]. In other words, ULK1 is a vital molecule to promote autophagy and is stimulated by AMPK and inhibited by the $\mathrm{PI} 3 \mathrm{~K} / \mathrm{AKT} / \mathrm{mTOR}$ pathway. In addition, it is worth mentioning that AMPK has a regulatory effect on the PI3K/Akt/mTOR pathway, that is, these pathways and molecules are closely related to this control and act directly on autophagy [34,38]. Thus, it can be concluded that both the inflammatory process and the autophagic process interact with each other and are points that are significantly affected in CKD. Therefore, these routes must be considered in this approach, mainly so that an adequate intervention can be proposed.

One of the factors that regulate the inflammatory and autophagic process is exercise, which has been studied as a form of non-drug treatment for various diseases, acting in the reduction of cardiovascular risk, in the regulation of blood pressure, in increasing insulin sensitivity, in improving cachexia, in regulating pressure arterial, in controlling anxiety, in reducing inflammation - including by stimulating M2 macrophages, in the control of non-alcoholic fatty liver disease, in the control of type 2 diabetes mellitus in addition to acting in the neurological function. In this sense, physical exercise has also been proposed as an alternative/complementary treatment for CKD. Thus, exercise can act in several ways: in controlling inflammation and in controlling autophagy [37,39-46]. In this way, physical exercise has also been proposed as an alternative/complementary treatment in cases of CKD, for example by modulating the inflammatory and autophagic processes. Regarding the inflammatory process, exercise has been shown to be efficient in promoting the release of IL- 6 by the skeletal muscle (myocin), which promotes the release of IL-10, the main cytokine with anti-inflammatory function, especially during exercise resisted/moderate [47]. In addition, both aerobic and anaerobic exercise are able to promote a reduction in inflammation by activating AMPK and mTOR respectively, both molecules capable of promoting the conversion of M1 (inflammatory) macrophages to M2 (anti-inflammatory) [31,36].

Regarding the autophagic process, physical activity is able to act in different ways depending on the type of exercise. Aerobic exercises stimulate autophagy already resisted anaerobic exercises, trigger the inhibition of autophagy, thus taking into account in view of this marked autophagic activation the most appropriate in this context is to establish moderate resistance training for this patient with CKD and SARS-CoV2 infection. But in any case, it is important to point out how each type of exercise contributes differently to the autophagic process, since ROS are able to relate to these molecules and modulate the process [24,48,49]. In aerobic exercise, the increase in adenosine monophosphate (AMP) and adenosine diphosphate (ADP) activates AMPK which in turn instead, it acts by inhibiting the PI3K/AKT/mTOR pathway, in addition to activating the ULK1 complex, thus stimulating autophagy $[34,37,50]$. In the resistance exercise, there is an increase in the Growth Factor similar to insulin-1 (IGF-1), this is recognized by the IGF-1 receptors (IGF$1 R$ ) and activate $\mathrm{PI} 3 \mathrm{~K}$, thus, the product of this reaction is phosphatidylinositol-3,4,5triphosphate (PI3P), which acts as a second messenger that recruit AKT, this in turn activates mTOR, completing the sequence of the $\mathrm{PI3K} / \mathrm{AKT} / \mathrm{mTOR}$ pathway, which, in turn, as previously mentioned, manages to inhibit ULK1 and therefore, can inhibit also autophagy (Fig. 2) $[34,51,52]$.

The example of treadmill aerobic exercise consumes many nutrients and energy cells, mainly ATP, when it is used it releases adenosine monophosphate (AMP) that activates AMPK, which in turn inhibits the PI3K/Akt/mTOR pathway and activates the ULK1 complex, stimulating autophagy. In the patient in the image above, a resistance exercise is represented - a merely illustrative extension chair -, in this picture, the resistance exercise activates IA-PI3K by growth factors, mainly with IGF-1, which is recognized by IGF-1R, this, for its instead, it activates Akt via PI3P, then Akt activates mTOR which inhibit the ULK1 complex and therefore autophagy. In addition, an anti-inflammatory action of exercise can also be perceived by promoting the release of IL-6, which stimulates the release of $\mathrm{IL}-10$, widely known for its antiinflammatory power. 


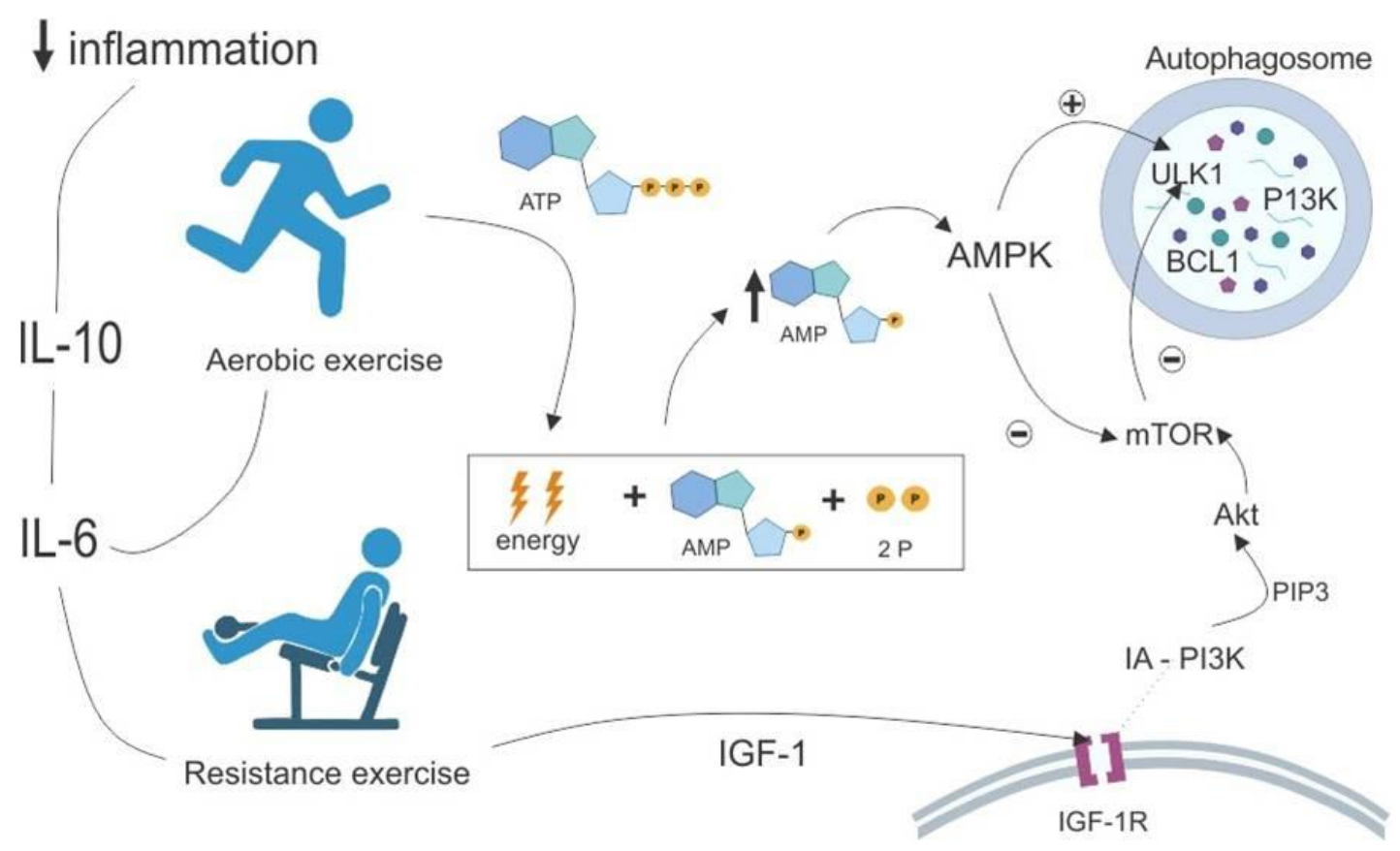

Fig. 2. Representation of the influence of aerobic exercise and resistance in the autophagic pathways and its result in the autophagosome

Source: The authors, 2020

AMP: adenosine monophosphate; ATP: adenosine triphosphate; AMPK: AMP-activated protein kinase; PI3K: Phosphoinositide 3-kinase; Akt or PKB: protein kinase B; mTOR: mammalian target of rapamycin; ULK1: Autophagy activation kinase 1 similar to Unc-51; BCL1: beclin 1; PI3K: Phosphoinositide 3-kinase; IA-PI3K: Class A 3-kinase phosphoinositide; P: phosphate; IGF-1: insulin-like growth factor 1; IGF-1R: Receptor for insulin-like growth factor 1; PI3P: Phosphatidylinositol-3-phosphate; IL-6: Interleukin 6; IL-10: Interleukin 10

\section{THERAPEUTIC POTENTIAL OF EXERCISE IN CKD AND COVID-19}

Individuals, affected with CKD have less physical function, as well as impaired physical performance, thus contributing to a situation of high motor disability, fragility and consequently, increased risk of mortality. Thus, the regular practice of physical exercises contributes to improvements in motor functions, in order to improve the efficiency of the mitochondrial oxidative metabolism of the muscle, as well as improvements in the immune system, promoting a framework of reduction of systemic inflammation [53].

Physical exercise, especially for the population with renal impairment, is essential, since it acts to improve sarcopenia, physical performance, quality of life, physical function and immunity. The exercise in question does not refer to running marathons, but simple walks are enough to achieve the benefits mentioned. Studies have shown that walking regularly has reduced the number of hospitalizations in patients with CKD.
It is important to note that there are physiological limitations of individuals with CKD regarding the performance of physical activities, since these people generally have associated comorbidities. According to the National Kidney Disease Outcomes Quality Initiative (KDOQI), patients with CKD should be evaluated according to their particularities [53]. Therefore, the recommendations regarding the exercises are: aerobic exercises (walking, swimming and cycling) should be performed at moderate intensity, two to three times a week; resistance exercises (multiarticular) should also be performed at moderate intensity and twice a week; flexibility exercises (combination of aerobic and resistance exercises) should be performed at low intensity with a frequency of five times a week, ten minutes a day [53].

Regarding the immune system, it is important to consider the inflammatory condition of patients with CKD. The inflammatory process is responsible for causing damage to the renal tubules, thus compromising renal function. Thus, inflammation provides an inflammatory response 
that, in turn, can progress to renal fibrosis [33]. Hence, physical exercise acts in modulating the immune system, in order to strengthen "immune surveillance", since it promotes an increase in the circulation of anti-inflammatory cytokines and immunoglobulins. The exercise acts on cytokines, neutrophils, leukocytes, antigen presenting cells and lymphocytes. With regard to cytokines, exercise is able to promote the release of interleukin-6 (IL-6), responsible for stimulating the synthesis of IL-1 and IL-10, which have anti-inflammatory action. Regarding neutrophils, it appears that, after exercise, there is a reduction in neutrophil chemotaxis without compromising bactericidal activity. Regarding leukocytes, leukocytosis is observed after physical exercise. Antigen-presenting cells reduce the presentation of antigens to $\mathrm{T}$ lymphocytes, thereby decreasing the Th-1 inflammatory response, thereby preventing tissue damage. Finally, during moderate exercise, an increase in TCD4+ lymphocytes occurs, contributing to the prevention of infections by intracellular microorganisms [1]. In addition, macrophages and dendritic cells act as antigen presenting cells for lymphocytes through a major histocompatibility complex, in order to provide an environment of cytokines, which will act as activators of the immune system. Lymphocytes play a fundamental role against viral microorganisms, since TCD8 + lymphocytes are responsible for direct toxicity to cells infected by the virus. In line, TCD4 + lymphocytes stimulate the production of neutralizing antibodies through the activation of B lymphocytes. In addition, there is a recruitment process for neutrophils and monocytes in order to promote other ways of amplifying the immune response. This process occurs through the action of auxiliary $T$ lymphocytes, especially Th-1 [54,55].

Recently, evidence has emerged that directly links autophagy with CKD, elucidating its essential role in this disease. Its action on healthy kidneys has great amplitude, being able to regulate the production of extracellular matrix and also act as a protective factor against necrosis and apoptosis during situations of cellular stress in mesangial cells thus avoiding necrosis and suppression of the foot processes through the mTOR pathway, with autophagy being the protagonist in maintaining renal function. Furthermore, autophagy acts in maintaining homeostasis in renal endothelial cells and also in combating toxic agents, proteins and damaged DNAs [29,56-60]. However, autophagy has a negative effect on CKD, as it is above the normal rate in skeletal muscle, causing loss of lean mass and mitochondrial dysfunction of muscle cells. It has also been shown that inhibition of autophagy reduces the loss of skeletal muscle cells, making it essential to know the ways in which physical exercise can modulate autophagy in patients with CKD [61]. Thus, anaerobic exercise promotes activation via mTOR and consequently, decreases autophagy, since the promotion of the PI3K/AKT/mTOR pathway inhibits ULK1. Consequently, regular physical exercise is able to reduce the expression of IC3, which is a marker of autophagosome formation and consequently, mitigate excessive autophagy, reducing the loss of skeletal muscle tissue, showing the importance of this mechanism [34,51].

Although information about the role of physical exercise in modulating autophagy often seems contradictory, studies have led to believe in the ability to maintain a dynamic balance promoted by physical exercise. This is because in individuals affected by CKD where autophagy was elevated, aerobic exercise reduced autophagic expression, while in a normal situation, exercise could increase it $[34,48]$.

\section{CONCLUSION}

The COVID-19 pandemic due to SARS-CoV-2 was responsible for compromising society, both in economic aspects and in relation to health and quality of life. Although the virus mainly affects the respiratory tissue, it is known that there are deleterious impacts on other organs and tissues. Individuals with associated comorbidities, including CKD, are more likely to develop the severe form of the disease. Thus, the regular practice of physical exercise is presented as a measure to assist in coping with COVID-19, since it provides major physiological changes that benefit the body.

Physical exercise is responsible for modulating the immune response, in order to enhance the organic defense mechanism by increasing the circulation of anti-inflammatory cytokines and immunoglobulins and consequently, increasing the body's anti-pathogenic activity. In addition, physical activity is also capable of modulating the autophagy process in chronic renal patients, given that autophagy has a renal protection effect against inflammatory stress resulting from CKD. Thus, physically active individuals, including the elderly and people with chronic diseases, are more likely to have a mild 
progression in relation to viral infections, including COVID-19.

\section{COMPETING INTERESTS}

Authors have declared that no competing interests exist.

\section{REFERENCES}

1. Da Silveira MP, Da Silva Fagundes KK, Bizuti MR, Starck E, Rossi RC, Silva DTR. Physical exercise as a tool to help the immune system against COVID-19: An integrative review of the current literature. Clinical and Experimental Medicine; 2020. Available:https://doi.org/10.1007/s10238020-00650-3.

2. Kunutsor SK, Laukkane JA. Renal complications in COVID-19: A systematic review and meta-analysis. Annals of Medicine; 2020.

Available:https://doi.org/10.1080/0785389. 2020.1790643.

3. Oyelade T, Alqahtani J, Canciani G. Prognosis of COVID-19 in patients with liver and kidney diseases: An early systematic review and meta-analysis. Tropical Medicine and Infectious Disease; 2020.

Available:https://doi.org/10.3390/tropicalm ed5020080.

4. Lin TA, Wu VCC, Wang CY. Autophagy in Chronic Kidney Diseases. Cells. 2019;8:120.

5. Clinical practice guidelines for chronic kidney disease: Evaluation, classification and stratification. National Kidney Foundation. 2002;39:1-356.

6. Bastos MG, Bregman R, Kirsztajn GM. Chronic kidney disease: Frequent and severe, but also preventable and treatable. Journal of the Brazilian Medical Association. 2010;56:248-253.

7. Wrapp D, Wang N, Corbett KS, Goldsmith JA, Hsieh CL, Abiona O, et al. Cryo-EM structure of the 2019-nCoV spike in the prefusion conformation. Science. 2020; 367:1260-1263.

8. Diao B, Feng $Z$, Wang $C$, Wang $H$, Liu L, Wang $C$, et al. Human kidney is a target for novel severe acute respiratory syndrome coronavirus 2 (SARS-CoV-2) Infection. medRxiv; 2020.

Available:https://doi.org/10.1101/2020.03.0 4.20031120.
9. Brazil. Ministry of Health. 12/3: World Kidney Day. Virtual Health Library; 2020.

10. Zou X, Chen K, Zou J, Han P, Hao J, Han Z. Single-cell RNA-seq data analysis on the receptor ACE2 expression reveals the potential risk of different human organs vulnerable to 2019-nCoV infection. Front Med. 2020;14:185-192.

11. Martinez Rojas MA, Vega Vega $O$, Bobadilla NA. Is the kidney a target of SARS-CoV-2? Am J Physiol Renal Physiol. 2020;318:1454-1462.

12. Wang $\mathrm{XH}$, Mitch WE. Mechanisms of muscle wasting in chronic kidney disease. Nat Rev Nephrol. 2014;10:504-516.

13. Mihai S, Codrici E, Popescu ID, Enciu AM, Albulescu L, Necula LG, et al. Inflammation-Related Mechanisms in Chronic Kidney Disease Prediction, Progression, and Outcome. J Immunol Res. 2018;2018:2180373.

14. Qian Q. Inflammation: A key contributor to the genesis and progression of chronic kidney disease. Contributions to Nephrology. 2017;191:72-83.

15. Imig JD, Ryan MJ. Immune and inflammatory role in renal disease. Compr Physiol. 2013;3:957-976.

16. Pan XW, Xu D, Zhang $H$, Zhou W, Wang $\mathrm{LH}$, Cui XG. Identification of a potential mechanism of acute kidney injury during the COVID-19 outbreak: A study based on single-cell transcriptome analysis. Intensive Care Med. 2020;46:1114-1116.

17. Adapa S, Chenna A, Balla M, Merugu GP, Koduri MN, Daggubati SR, et al. COVID-19 pandemic causing acute kidney injury and impact on patients with chronic kidney disease and renal transplantation. J Clin Med Res. 2020;12:352-361.

18. Huang C, Wang Y, Li X, Ren L, Zhao J, Hu $Y$, et al. Clinical features of patients infected with 2019 novel coronavirus in Wuhan, China. Lancet. 2020;395:497-506.

19. Arentz M, Yim E, Klaff L, Lokhandwala S, Riedo FX, Chong M, et al. Characteristics and outcomes of 21 critically III patients With COVID-19 in Washington State. JAMA. 2020;323:1612-1614.

20. Guan WJ, Ni ZY, Hu Y, Liang WH, Ou CQ, $\mathrm{He} J X$, et al. Clinical characteristics of coronavirus disease 2019 in China. N Engl J Med. 2020;382:1708-1720.

21. Cheng $Y$, Luo $R$, Wang $K$, Zhang M, Wang $Z$, Dong L, et al. Kidney impairment is associated with in-hospital death of COVID-19 patients. medRxiv; 2020. 
Available:https://doi.org/10.1101/2020.02.1 8.20023242

22. Chawla LS, Eggers PW, Star RA, Kimmel PL. Acute kidney injury and chronic kidney disease as interconnected syndromes. $\mathrm{N}$ Engl J Med. 2014;371:58-66.

23. Li Z, Wu M, Yao J, Guo J, Liao X, Song S, et al. Caution on kidney dysfunctions of COVID-19 patients. Med Rxiv; 2020.

Available:https://doi.org/10.1101/2020.02.0 8.20021212.

24. Wang L, Wang J, Cretoiu D, Li G, Xiao J. Exercise-mediated regulation of autophagy in the cardiovascular system. Journal of Sport and Health Science. 2020;9:203210.

25. Sekar RR, Patil D, Baum Y, Pearl J, Bausum A, Bilen MA, et al. A novel preoperative inflammatory marker prognostic score in patients with localized and metastatic renal cell carcinoma. Asian Journal of Urology. 2017;4:230-238.

26. Vivar AR, Finke JH, Bukowski R. The role of inflammation in kidney cancer. Advances in Experimental Medicine and Biology. 2014;816:197-234.

27. Apanovich NV, Peters MV, Apanovich PV, Kamolov BS, Matveev VB, Ginter EK, et al. Expression profiles of genes-potential therapy targets-and their relationship to survival in renal cell carcinoma. Doklady Biochemistry and Biophysics. 2018;478: 14-17.

28. Dungey M, Hull KL, Smith AC, Burton JO, Bishop NC. Inflammatory factors and exercise in chronic kidney disease. International Journal of Endocrinology. 2013;2013:1-13.

29. Lin TA, Wu VCC, Wang CY. Autophagy in Chronic Kidney Diseases. Cells. 2019; 8:61.

30. Henry BM, De Oliveira MHS, Benoit S, Plebani M, Lippi G. Hematologic, biochemical and immune biomarker abnormalities associated with severe illness and mortality in coronavirus disease 2019 (COVID-19): A meta-analysis. Clinical Chemistry and Laboratory Medicine (CCLM). 2020;58:1021-1028.

31. Han Y, Liu Y, Zhao Z, Zhen S, Chen J, Ding $N$, et al. Does physical activity-based intervention Improve systemic proinflammatory cytokine levels in overweight or obese children and adolescents? Insights from a MetaAnalysis of Randomized Control Trials. Obes Facts. 2019;12:653-668.
32. Mukhopadhyay $\mathrm{P}$, Eid N, Abdelmegeed MA, Sen A. Interplay of oxidative stress, inflammation, and autophagy: Their role in tissue injury of the heart, liver, and kidney. Oxidative Medicine and Cellular Longevity. 2018;2018:1-3.

33. Kimura T, Isaka $\mathrm{Y}$, Yoshimori $\mathrm{T}$. Autophagy and kidney inflammation. Autophagy. 2017;13:997-1003.

34. Zhang T, Guo J, Gu J, Chen K, Li H, Wang J. Protective Role of mTOR in liver ischemia/reperfusion injury: involvement of inflammation and autophagy. Oxidative Medicine and Cellular Longevity. 2019; 2019:1-17.

35. Sureshbabu A, Ryter SW, Choi ME. Oxidative stress and autophagy: Crucial modulators of kidney injury. Redox Biology. 2015;4:208-214.

36. Cheng AW, Tan X, Sun JY, Gu CM, Liu C, Guo $X$. Catechin attenuates TNF- $\alpha$ induced inflammatory response via AMPKSIRT1 pathway in 3T3-L1 adipocytes. PLoS ONE. 2019;14:0217090.

37. Brandt N, Gunnarsson TP, Bangsbo J, Pilegaard $H$. Exercise and exercise training-induced increase in autophagy markers in human skeletal muscle. Physiol Rep. 2018;6:13651.

38. Jia J, Abudu YP, Claude Taupin A, Gu Y, Kumar S, Choi SW, et al. Galectins control MTOR and AMPK in response to lysosomal damage to induce autophagy. Autophagy. 2019;15:169-171.

39. Sharman JE, La Gerche A, Coombes JS. Exercise and cardiovascular risk in patients with hypertension. American Journal of Hypertension. 2015;28:147-158.

40. Dimeo F, Pagonas N, Seibert F, Arndt R, Zidek W, Westhoff TH. Aerobic exercise reduces blood pressure in resistant hypertension. Hypertension. 2012;60:653658.

41. Park YM, Myers M, Vieira Potter VJ. Adipose tissue inflammation and metabolic dysfunction: Role of exercise. Mo Med. 2014;111:65-72.

42. Pescatello LS, Buchner DM, Jakicic JM, Powell KE, Kraus WE, Bloodgood B, et al. Physical activity to prevent and treat hypertension: A systematic review. Medicine \& Science in Sports \& Exercise. 2019;51:1314-1323.

43. LeBouthillier DM, Asmundson GJG. The efficacy of aerobic exercise and resistance training as transdiagnostic interventions for anxiety-related disorders and constructs: A 
randomized controlled trial. Journal of Anxiety Disorders. 2017;52:43-52.

44. Hashida R, Kawaguchi T, Bekki M, Omoto $M$, Matsuse $H$, Nago $T$, et al. Aerobic vs. resistance exercise in non-alcoholic fatty liver disease: A systematic review. Journal of Hepatology. 2017; 66:142-152.

45. Nery C, Moraes SRAD, Novaes KA, Bezerra MA, Silveira PVDC, Lemos A. Effectiveness of resistance exercise compared to aerobic exercise without insulin therapy in patients with type 2 diabetes mellitus: A meta-analysis. Brazilian Journal of Physical Therapy. 2017;21:400-415.

46. Hayek LE, Khalifeh M, Zibara V, Assaad $\mathrm{RA}$, Emmanuel N, Karnib N, et al. Lactate mediates the effects of exercise on learning and memory through SIRT1dependent activation of hippocampal brainderived neurotrophic factor (BDNF). J Neurosci. 2019;39:2369-2382.

47. Santos GC, Faria WF, Sasaki J, Elias RMG, Neto AS. Acute effects of physical exercise at different intensities on inflammatory markers in obese adolescents. J Physical Edu. 2019;30: 3014.

48. Yan Y, Zhou X, Xu H, Melcher K. Structure and physiological regulation of AMPK. Int $\mathrm{J}$ Mol Sci. 2018;19:3534.

49. Alers S, Loffler AS, Wesselborg S, Stork B. Role of AMPK-mTOR-Ulk1/2 in the regulation of autophagy: Cross talk, shortcuts and feedbacks. Molecular and Cellular Biology. 2012;32:2-11.

50. Wu NN, Tian H, Chen P, Wang D, Ren J, Zhang Y. Physical exercise and selective autophagy: Benefit and risk on cardiovascular health. Cells. 2019;8:1436.

51. Agostini D, Natalucci V, Baldelli G, Santi MD, Zeppa SD, Vallorani L, et al. New insights into the role of exercise in inhibiting mTOR signaling in triple-negative breast cancer. Oxidative Medicine and Cellular Longevity. 2018;2018:1-19.
52. Yuan TL, Cantley LC. PI3K pathway alterations in cancer: variations on a theme. Oncogene. 2008;27:5497-5510.

53. Roshanravan B, Gamboa J, Wilund K. Exercise and CKD: Skeletal muscle dysfunction and practical application of exercise to prevent and treat physical impairments in CKD. Am J Kidney Dis. 2017;69:837-852.

54. Prompetchara E, Ketloy C, Palaga T. Immune responses in COVID-19 and potential vaccines: lessons learned from SARS and MERS epidemic. Asian Pac J Allergy Immunol. 2020;38:1-9

55. Li G, Fan Y, Lai Y, Han T, Li Z, Zhou P, et al. Coronavirus infections and immune responses. J Med Virol. 2020;92:424-32.

56. Deng X, Xie Y, Zhang A. Advance of autophagy in chronic kidney diseases. Renal Failure. 2017;39:306-313.

57. Ding Y, Kim JK, Kim SI, Na HJ, Jun SY, Lee SJ, et al. TGF- $\beta 1$ protects against mesangial cell apoptosis via induction of autophagy. Journal of Biological Chemistry. 2010;285:37909-37919.

58. Wang Z, Choi ME. Autophagy in kidney health and disease. Antioxidants and redox signaling. 2014;20:519-537.

59. Andrade L, Rodrigues CE, Gomes SA, Noronha IL. Acute kidney injury as a condition of renal senescence. Cell transplantation. 2018;27:739-753.

60. Hsu YJ, Hsu SC, Huang SM, Lee HS, Lin $\mathrm{SH}$, Tsai CS, et al. Hyperphosphatemia induces protective autophagy in endothelial cells through the inhibition of Akt/mTOR signaling. Journal of Vascular Surgery. 2015;62:210-221.

61. Aniort J, Stella A, Philipponnet C, Poyet A, Polge C, Claustre A, et al. Muscle wasting in patients with end-stage renal disease or early-stage lung cancer: common mechanisms at work. Journal of Cachexia, Sarcopenia and Muscle. 2019;10:323-337.

(c) 2021 Bizuti et al.; This is an Open Access article distributed under the terms of the Creative Commons Attribution License (http://creativecommons.org/licenses/by/4.0), which permits unrestricted use, distribution, and reproduction in any medium, provided the original work is properly cited.

Peer-review history:

The peer review history for this paper can be accessed here: http://www.sdiarticle4.com/review-history/66103 